\title{
Plataforma Online de Evaluación de Compromiso Escolar, Versión 2.0: Desde la Experiencia Chilena al Uso en Países de Iberoamérica
}

\author{
Online Platform for the Evaluation of School Engagement, 2.0 Version: From the \\ Chilean Experience to its Use in Iberoamerican Countries
}

\author{
Mahia Saracostti ${ }^{1}$, Belén Sotomayor ${ }^{2}$, María Teresa Hernández ${ }^{3}$, Laura Lara ${ }^{4}$, Edgardo Miranda-Zapata ${ }^{5}$, \\ José Aparicio $^{6}$, Rosa María Diaz-Jiménez ${ }^{7}$, Fernando Acevedo ${ }^{8}$ y Sergio Dominguez-Lara ${ }^{9}$
}

\begin{abstract}
Resumen
Este artículo presenta el desarrollo de la versión 2.0. de una plataforma tecnológica de evaluación del compromiso escolar utilizada en Chile, Colombia, Perú, Uruguay y España. La plataforma alberga instrumentos de medición del compromiso escolar y los factores contextuales, informes de resultados y recursos educativos. Lo cual tiene como objetivo acompañar las trayectorias educativas, entregar apoyos oportunos y promover la retención escolar. El desarrollo de la plataforma ha sido incremental basado en proyectos I+D asociados. Ha sido reconocida por la Cátedra UNESCO sobre Niñez/Juventud, Educación y Sociedad y ofrece la oportunidad de ser utilizada en otros países, en un momento donde el abandono escolar es una preocupación de especial relevancia a nivel latinoamericano y global.
\end{abstract}

Palabras clave: plataforma tecnológica, compromiso escolar, factores contextuales, trayectorias educativas, plataforma web

\footnotetext{
Abstract

This article presents the development of version 2.0 of a technological platform for evaluating school engagement in Chile, Colombia, Peru, Uruguay and Spain. The platform contains instruments for measuring school engagement and contextual factors, reports of results, and educational resources. This aims to accompany educational paths, provide timely supports, and promote school retention. The development of the platform has been incremental based on associated R\&D projects. It has been recognized by the UNESCO Chair in Children, Youth, Education and Society and it offers the opportunity to be used in other countries, at a time when school dropout is a concern of special relevance in Latin American and global level.

Keywords: technological platform, school engagement, contextual factors, educational trajectories, web platform

${ }^{1}$ Doctora en Social Welfare. Profesora Titular de la Escuela de Trabajo Social de la Facultad de Ciencias Sociales. Directora de la Cátedra UNESCO Niñez, Juventud, Educación y Sociedad. Universidad de Valparaíso. Universidad de Valparaíso, Blanco 951, Valparaíso, Chile. Tel.: +56 998293161. Correo: mahia.saracostti@uv.cl

${ }^{2}$ Máster en Educación. Investigadora. Universidad de la Frontera, Chile. Estudiante PhD Educación, Universidad de Almería, España. Avenida Francisco Salazar, 01145, Temuco, Chile. Tel.: +56 952531464. Correo: mbsotomayor@uc.cl

${ }^{3}$ Magister en Educación. Investigadora. Universidad de la Frontera. Universidad de la Frontera, Avenida Francisco Salazar, 01145, Temuco, Chile. Tel.: +56 979778350. Correo: maitehernandezy@gmail.com

${ }^{4}$ Laura Lara. Doctora en Psicología. Profesora Titular. Universidad Autónoma de Chile, 5 poniente 1670, Talca, Chile. Tel.: +56 712735770. Correo: 1larav@uautonoma.cl

${ }^{5}$ Doctor en Metodología de las Ciencias del Comportamiento y de la Salud. Académico e Investigador. Núcleo Científico Tecnológico en Ciencias Sociales y Humanidades, Universidad de La Frontera. Universidad de la Frontera, Avenida Francisco Salazar, 01145 . Temuco, Chile. Tel.: +56 452596732. Correo: edgardo.miranda@ufrontera.cl

${ }^{6}$ Doctor en Psicología. Decano del Instituto de Estudios en Educación. Universidad del Norte. Universidad del Norte. Km.5 Vía Puerto Colombia, Barranquilla, Colombia. Tel.: +57 53509799. Correo: aparicio@uninorte.edu.co

7 Doctora en Ciencias Sociales. Profesora Titular de Trabajo Social y Servicios Sociales. Universidad Pablo de Olavide. Facultad de Ciencias Sociales, Universidad Pablo de Olavide de Sevilla, Ctra. de Utrera 1, CP. 41013 Sevilla, España. Tel.: +34 610125899, Correo: rdiajim@upo.es

${ }^{8}$ Doctor en Educación. Profesor Adjunto. Universidad de la República de Uruguay. Centro Universitario de Rivera, Ituzaingó 667, C.P. 40.000, Rivera, Uruguay. Tel.: +59899866115. Correo: face@ cur.edu.uy

${ }^{9}$ Doctor en Psicología. Docente-investigador. Universidad de San Martín de Porres y Universidad Privada San Juan Bautista. Av. Juan A Lavalle S/N, Lima 9, Perú. Tel.: +51 2545302. Correo: sergio.dominguez@upsjb.edu.pe
} 


\section{Introducción}

El compromiso escolar (CE) es una variable clave para prevenir temprana y oportunamente la desescolarización y promover la retención escolar en el sistema educativo (Christenson et al., 2008). Investigadores provenientes de distintas áreas (Ashwin \& McVitty, 2015; Bowles et al., 2013; Fredricks, Filsecker, \& Lawson, 2016; Fredricks, Reschly, \& Christenson, 2019; Miranda-Zapata, Lara, Navarro, Saracostti, \& de-Toro, 2018; Wang $\&$ Fredricks, 2014) concuerdan en que el CE es un factor clave en la desescolarización, ya que el abandono no suele ser un acto repentino, sino que constituye la etapa final de un proceso dinámico y acumulativo de pérdida de compromiso con los estudios y de vinculación con el sistema escolar que, desde una dimensión relacional o colectiva, reflejaría un hito extremo de exclusión socioeducativa.

El CE también es un predictor estadísticamente significativo de las variables que intervienen en el éxito de las trayectorias educativas (Archambault, Janosz, Fallu, \& Pagani, 2009; Zaff et al., 2017), reflejándose en resultados tales como el rendimiento, asistencia, promoción entre niveles y ciclos educativos, graduación escolar, transición a la educación superior, y en el grado de involucramiento que tienen los estudiantes con su escuela y su quehacer educativo (Jimerson, Renshaw, Stewart, Hart, \& O'Malley, 2009; Roundfield, Sánchez, \& McMahon, 2016). Por otro lado, Csikszentmihalyi y Shernoff (2009), señalan que el CE estaría basado completamente en la experiencia de los estudiantes, por tanto, debería ser considerado como una "experiencia de aprendizaje", que tiene valor en sí misma, y no tan solo como un predictor (Fredrick et al., 2019).

En el contexto de este artículo, el CE se define como la participación activa del/la estudiante en las actividades académicas, curriculares o extracurriculares (Saracostti, Lara, \& MirandaZapata, 2019). Los y las estudiantes comprometidos consideran el aprendizaje como significativo y lo reflejan en la motivación y perseverancia que despliegan para su construcción, y que sirven de anclaje para futuras experiencias educativas. Al mismo tiempo, el CE se convierte en un referente para que otros estudiantes se sumen a dichas experiencias atraídos por la influencia multidimensional de la configuración del aprendizaje, dejando en evidencia que no sólo depende del esfuerzo individual, sino de un conjunto de elementos que forman parte del contexto, favoreciendo con ello el proceso de aprender (Christenson, Reschly, \& Wylie, 2012; Fredricks et al., 2016, 2019; González-González, 2010, Saracostti, et al., 2019). Se sabe que el $C E$ es una variable altamente influenciada por factores contextuales (FC). Estudios recientes apuntan a que familias, pares y profesorado son los tres contextos relacionales principales asociados con el involucramiento escolar (Ansong, Okumu, Bowen, Walker, \& Eisensmith, 2017; FernándezZabala, Goni, Camino, \& Zulaika, 2016; Quin, Heerde, \& Toumbourou, 2018). En definitiva, el CE no pone énfasis sólo en el estudiante como unidad de análisis, sino que considera aquellos elementos contextuales/relacionales, familiares y escolares que inciden en su compromiso y experiencia escolar, por lo que el contexto se configura en condición clave de la sociología de los procesos educativos.

La plataforma de evaluación online de compromiso escolar desarrollada y validada en Chile (y que ha servido de referente para otros países de Iberoamérica), operacionaliza el CE a partir de tres dimensiones principales: afectiva, conductual y cognitiva (Saracostti et al., 2019). La primera versión se inició desde una base web que posibilitaba la aplicación de cuestionarios online a estudiantes de séptimo básico a primero medio en Chile (en los que los y las estudiantes tienen entre 12 y 14 años, a excepción de los casos en los que repiten curso) y la extracción de reportes en línea (Saracostti et al., 2019). Con el fin de facilitar el análisis de los resultados, se diseñó una versión 2.0, logrando ampliar la cobertura de seguimiento desde los estudiantes de $7^{\circ}$ básico a $3^{\circ}$ medio. En ella, el/la profesor/a, los equipos psicosociales o psicopedagógicos, y directivos de cada Institución, a través de usuarios/as personalizados, pueden acceder a los resultados que arroja el sistema a nivel de estudiante, curso y colegio, y a las sugerencias para promover trayectorias educativas exitosas para cada estudiante. El objetivo de este cambio se debe a que no sólo es relevante detectar de manera temprana estudiantes 
que presentan un bajo $\mathrm{CE}$, sino que también es deseable prevenir que dicha falta de compromiso se vaya agudizando en el tiempo, por lo cual se hace necesario buscar formas de promover dicho compromiso en toda la comunidad estudiantil.

La plataforma versión 2.0 incluye un módulo de recursos educativos que permite dar apoyo a la interpretación de los resultados de las encuestas y orientar las acciones que debiese considerar el colegio para potenciar y/o remediar los resultados de los alumnos. Actualmente, se trabaja en transformar el módulo de recursos educativos en un módulo de capacitación online que busca generar un curso breve e interactivo que facilite el uso de la plataforma, la comprensión de los conceptos de compromiso escolar y factores contextuales y su relevancia en el contexto de la prevención temprana de la deserción. Este módulo tiene como finalidad promover el diseño de acciones que fomenten la retención escolar de todos y todas las estudiantes del ciclo básico y medio, a partir del acceso y comprensión de los resultados que son arrojados por la plataforma.

Para facilitar la aplicación autónoma de la plataforma en los establecimientos escolares, se incluyeron soportes automatizados tales como: registro de participantes, creación y asignación de códigos de acceso, registro de nuevos colegios y sostenedores participantes; y creación de perfiles de usuarios.

\section{Método}

Para el desarrollo de este proyecto, se aplicó la metodología de desarrollo incremental inspirada en métodos rápidos, siguiendo las guías propuestas por la metodología de desarrollo de software basada en SCRUM ${ }^{1}$, la cual considera la participación de tres actores claves: el cliente o contraparte (representado por el equipo técnico e investigadores donde se han albergado los proyectos de $\mathrm{I}+\mathrm{D}$ ejecutados), un responsable de desarrollo (representado por la empresa) y un equipo de validación y desarrollo (compuesto por profesionales de ambos equipos). Además, se cuenta con la retroalimentación desde los

\footnotetext{
${ }^{1}$ Más información en http://www.proyectosagiles.org
}

diferentes equipos de los establecimientos escolares y las entidades asociadas al proyecto.

Esta metodología plantea la división del proyecto en versiones incrementales de los productos de software, que, para este caso, son los módulos de la plataforma tecnológica para la medición y reporte del CE y los factores contextuales. Los prototipos funcionales de los módulos fueron desarrollados como parte del proyecto, y han sido revisados periódicamente por un equipo interno y externo. Este último fue el responsable de su aplicación en los establecimientos educativos, favoreciendo durante todo el proceso, la incorporación de mejoras continuas a la plataforma.

Para el desarrollo de los módulos propuestos para la plataforma tecnológica de medición y reporte online, se llevaron a cabo las siguientes etapas asociadas a la metodología de construcción incremental:

a. Definición de Requerimientos: En esta etapa se realizó el levantamiento preliminar de requerimientos para los módulos a desarrollar y posteriormente su especificación técnicafuncional. A partir de estas definiciones se elaboró un listado detallado de las funcionalidades requeridas, junto con la estimación del esfuerzo necesario para desarrollar cada componente funcional.

b. Diseño Funcional y Gráfico: En esta etapa se llevó a cabo el diseño detallado de la estructura y componentes funcionales de cada uno de los módulos de software que componen la plataforma tecnológica. Además, se realizó un proceso de revisión y actualización de los ambientes de desarrollo para la construcción de la plataforma. Con base en estas definiciones se elaboró una propuesta gráfica para los módulos, que fueran coherentes entre sí y con los instrumentos de medición desarrollados por el equipo técnico.

c. Desarrollo Incremental: En esta etapa se realizó la programación de los módulos de software propuestos para la plataforma tecnológica, bajo un modelo de desarrollo incremental de prototipos. En forma periódica se fue liberando un prototipo para su revisión por parte de los distintos actores involucrados. En esta fase fue clave la estructura de colaboración establecida entre el equipo técnico y el equipo de desarrollo. 
d. Pruebas y Correcciones Técnicas: Para asegurar la calidad de los módulos de software desarrollados, se contempló un proceso paralelo de revisión de aspectos técnicos funcionales y de usabilidad de los componentes. Esta etapa permitió recopilar, sistematizar y mejorar las deficiencias de la plataforma a través de pruebas de carga, pruebas con expertos y pruebas con usuarios reales.

e. Aplicación: Esta etapa contempló las distintas tareas $\mathrm{y}$ actividades orientadas a mantener operativa la plataforma durante los procesos de aplicación de los instrumentos en los establecimientos escolares. En particular, se contempló aplicaciones a nivel piloto de los instrumentos y a nivel masivo en establecimientos escolares de Chile, Colombia, España, Perú y Uruguay.

\section{Resultados}

La plataforma tecnológica versión 2.0, para la medición de CE y los factores contextuales, es un sistema Web disponible en www.compromisoescolar.com. Como muestra la Figura 1, la plataforma de evaluación de CE versión 2.0 incluye tres módulos: Encuestas, resultados y recursos educativos.

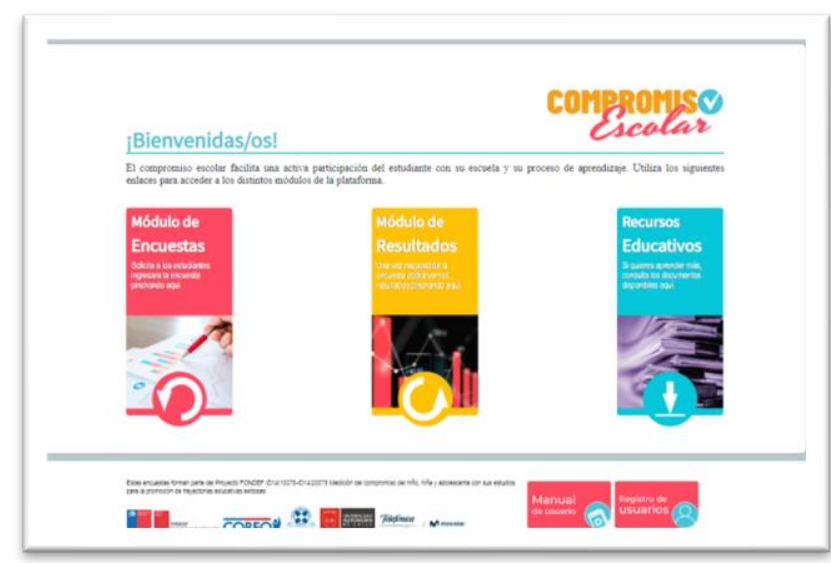

Figura 1. Página inicial de Plataforma online de evaluación de CE 2.0

Como muestra la Figura 2, los usuarios ingresan a la plataforma usando un código de autentificación creado y entregado previamente por la administración de la plataforma. Dependiendo del perfil de usuario del código asignado, los participantes tienen acceso a ciertas funcionalidades de los tres módulos de software definidos: el módulo de encuestas, el módulo de reportes y el módulo de apoyo pedagógico.

\section{1) Módulo de Encuestas}

El módulo de encuestas es un ambiente en el cual los participantes tienen acceso a la encuesta que deben responder, para posibilitar la medición del CE de los estudiantes y los factores contextuales que los afectan.

En particular, este módulo contempla una funcionalidad de autentificación por medio de una clave asignada previamente, que permite acceder a las encuestas en función de cada uno de los perfiles de los participantes: estudiantes, profesores/as y apoderados/as.

En el caso de los estudiantes, ellos ingresan usando el código asignado, que generalmente consiste en el RUT (Rol Único Tributario, DNI en España). La encuesta comienza con una página de bienvenida, en la cual se les motiva a completarla. Al presionar el botón de comenzar, se despliegan 12 pantallas continuas, con 6 ítems cada una, en los cuales deben seleccionar la opción de respuesta que más se acomode a su situación personal (Figura 3).

Como se muestra en la Figura 3, para facilitar la interacción con la encuesta, cada una de las pantallas incluye instrucciones, que se pueden desplegar o comprimir, una barra de avance que le indica al usuario en forma gráfica el porcentaje de llenado y botones para navegar a través de las pantallas de la encuesta. Además, incluye información visual frente a cada una de las opciones de respuesta para ayudar a la selección por parte de los estudiantes. Sin embargo, como una forma de asegurar que la encuesta sea respondida íntegramente por parte de los estudiantes se incorpora una funcionalidad que impide avanzar a la siguiente pantalla sin haber respondido todos los ítems. Al finalizar y enviar la encuesta, los estudiantes tienen acceso a un diploma de participación que puede ser descargado en PDF para su impresión o puede ser enviado a una dirección de correo electrónico.

\section{2) Módulo de Reportes}

Para facilitar el análisis e interpretación de los resultados que genera la aplicación para la medición del CE y los factores contextuales, se desarrolló un módulo de reportes que presenta en 


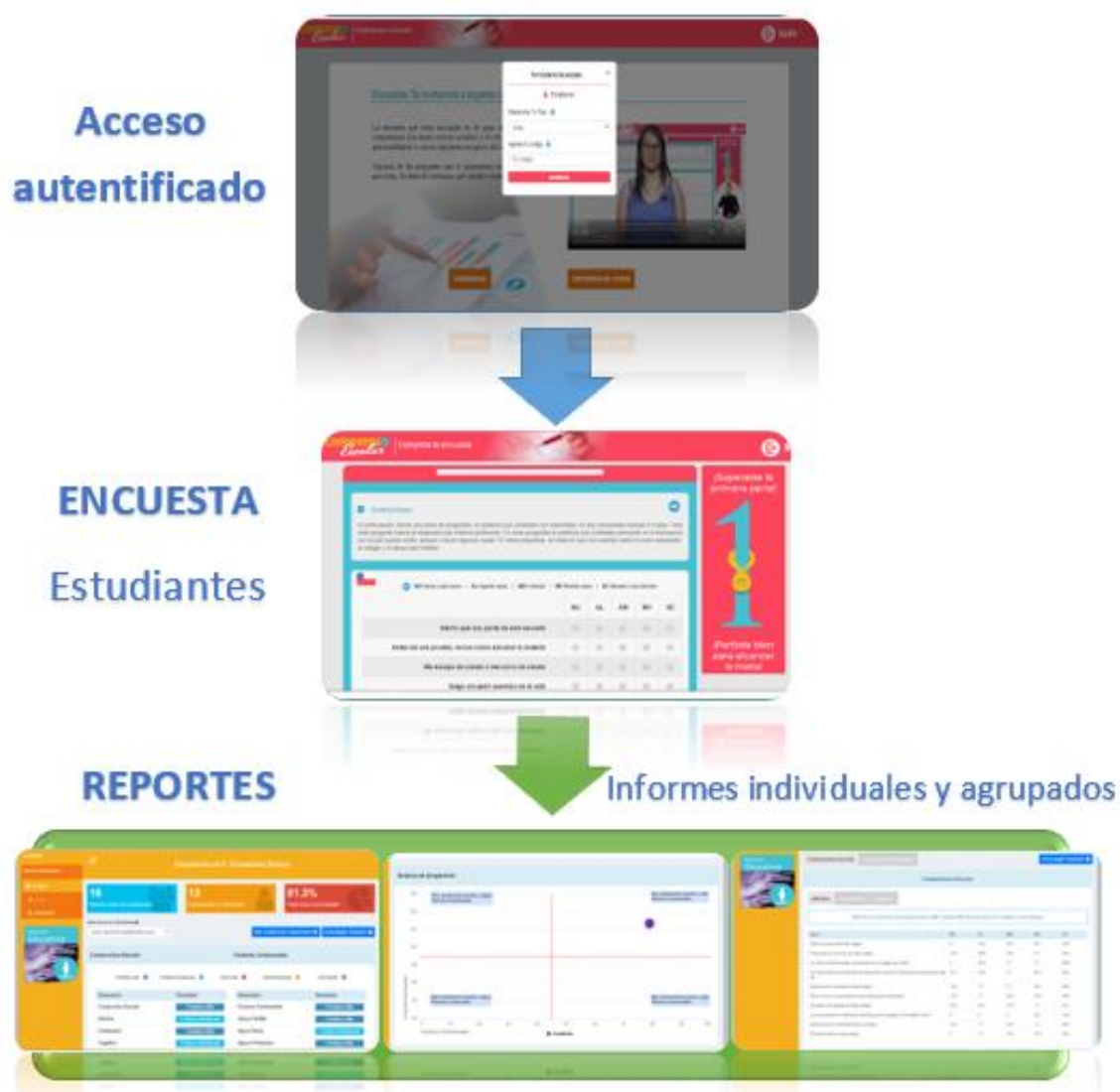

Figura 2. Plataforma Tecnológica

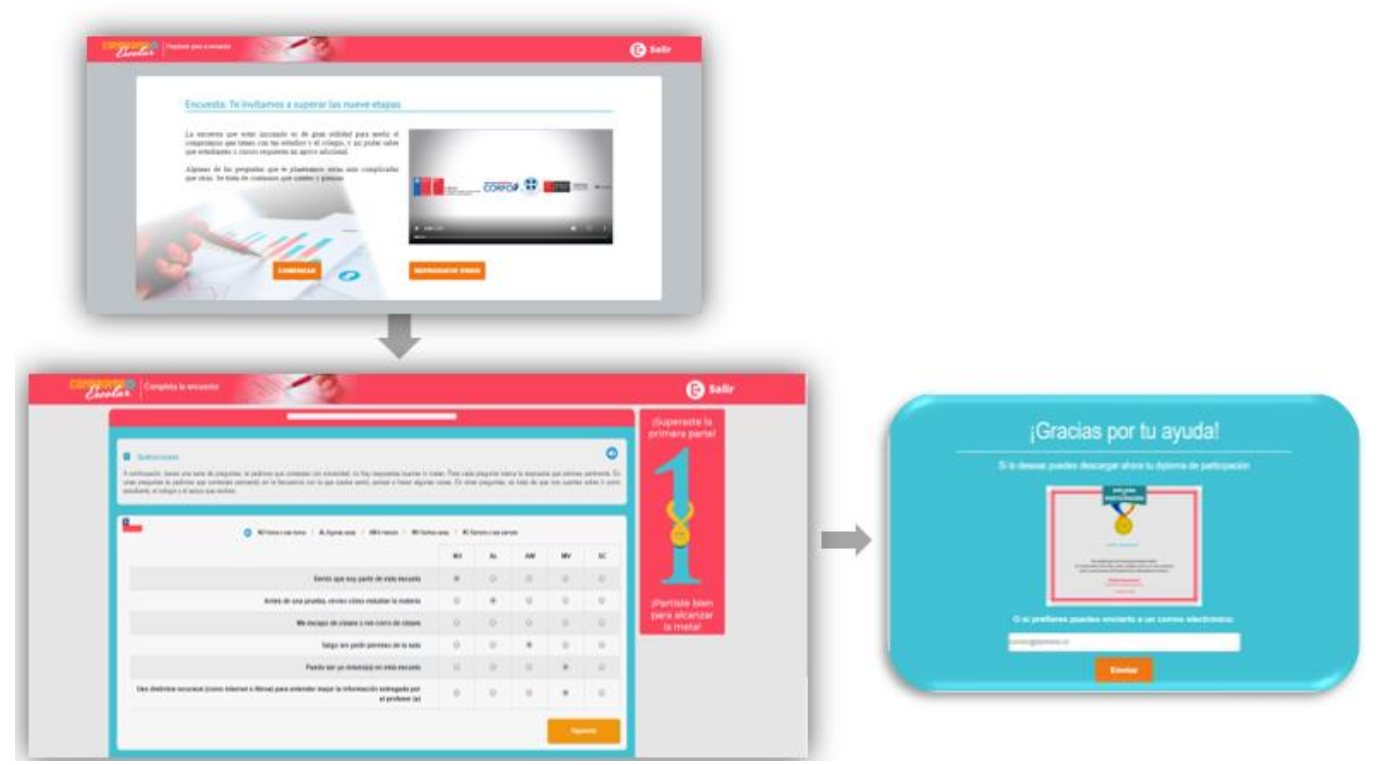

Figura 3. Encuesta de CE y los factores contextuales para Estudiantes

forma gráfica la medición: de forma individual, por curso, establecimiento y sostenedor. Para acceder a este módulo, se debe seleccionar un botón ubicado en la parte inferior del portal Web de compromiso escolar. Su ingreso está restringido a los usuarios que cuenten con una cuenta y clave creada por los administradores de la plataforma.

\section{Reportes para el Profesor}

Mediante este módulo el/la profesor/a tiene acceso a reportes individuales y grupales de sus estudiantes. En el caso del reporte individual, este corresponde a un conjunto de caracterizaciones cualitativas asociadas al puntaje obtenido por cada estudiante en las dimensiones del CE y los factores contextuales. Como se aprecia en la Figura 4, 

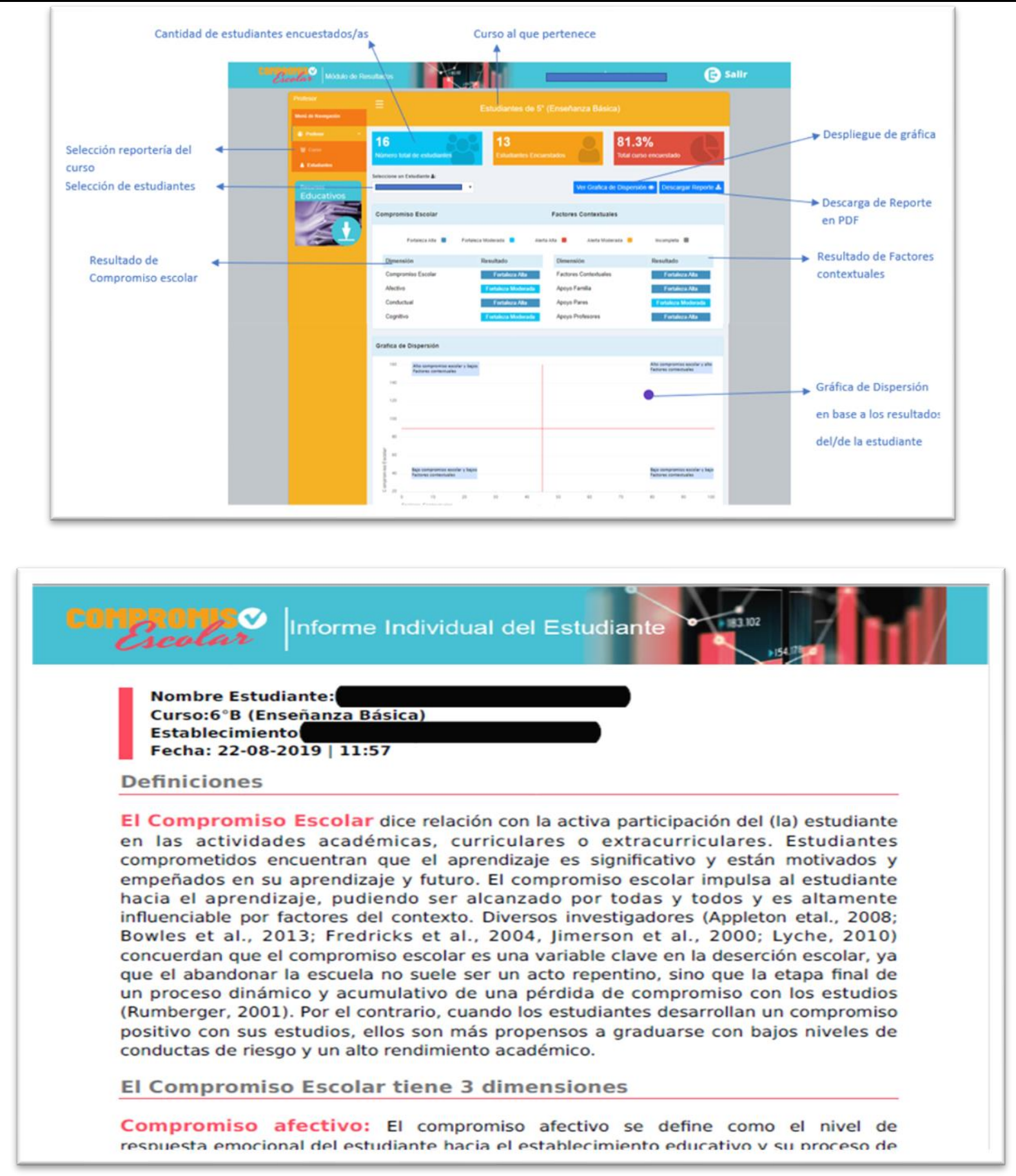

Figura 4. Reportes Individuales de Estudiantes web y descargable

Figura 4, el/la profesor/a y/o equipo de apoyo psicosocial o psicoeducativo (de acuerdo con la definición del equipo directivo del establecimiento escolar o sostenedor), luego de ingresar al módulo debe seleccionar la opción: estudiantes en el menú lateral izquierdo y seleccionar el estudiante sobre el cual desea revisar los resultados. A partir de esta selección se presentan un conjunto de fortalezas y alertas asociadas a cada dimensión del CE y los factores contextuales, las cuales incluyen un conjunto de caracterizaciones cualitativas asociados a cada estudiante. Nunca se entrega un puntaje de los estudiantes, ni se los clasifica o categoriza de ninguna forma. Tampoco se busca generar un semáforo de estudiantes con $\mathrm{CE}$ alto, medio o bajo. Lo que se pretende es identificar información relevante sobre las fortalezas y alertas de cada estudiante, proponiendo orientaciones específicas para promover el desarrollo del CE, a partir de la comprensión de los factores que inciden él, en especial, los de contexto o relacionales. 

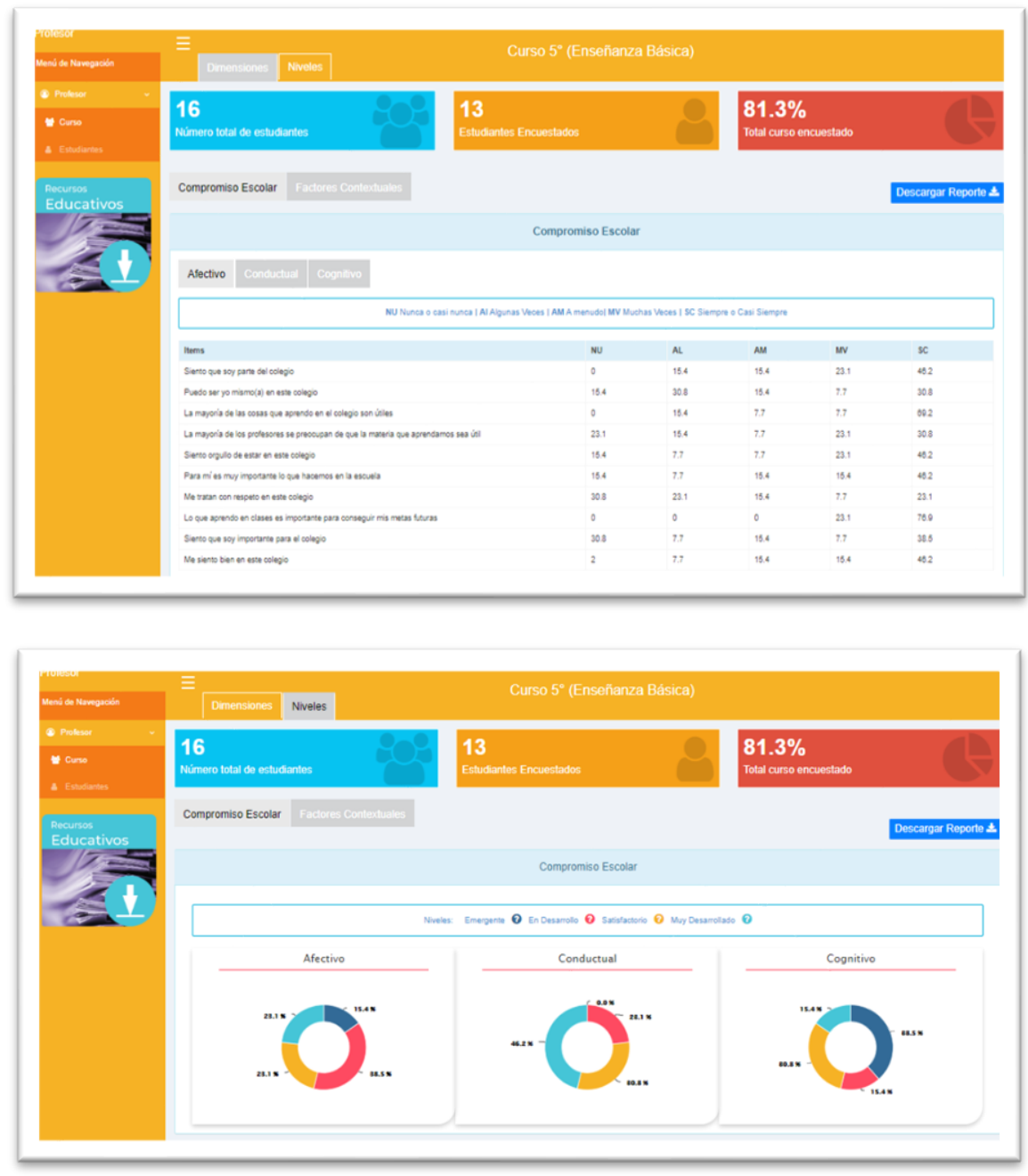

Figura 5. Reportes por Curso

El informe individual también está disponible en formato PDF, que puede ser descargado y revisado en forma impresa, como se muestra en la Figura 4.

En el caso de los reportes disponibles para los/as profesores/as o equipos de apoyos psicosociales o psicoeducativos, el módulo contempla dos tipos: de distribución de respuesta de los ítems y de niveles de desarrollo (Figura 5).

El reporte de dimensiones presenta la distribución porcentual de las opciones de respuesta que marcaron los estudiantes para cada uno de los ítems, que miden las tres dimensiones del CE y las tres dimensiones de los factores contextuales. Para revisar esta información el/la profesor/a debe seleccionar primero la variable que desea profundizar y luego seleccionar la dimensión sobre la cual quiere conocer la distribución de respuestas.

En el caso del reporte por niveles, este presenta los porcentajes de forma gráfica para cada dimensión, diferenciando cada uno de los cuatro niveles de desarrollo del CE y los factores contextuales. Los puntajes de corte de cada uno de los 4 niveles de desarrollo fueron definidos por el equipo de diseño de los instrumentos con base en una división teórica, en función de los valores máximos y mínimos de la escala.

\section{Reportes para el Colegio}

A nivel de colegio el módulo de reportes presenta información disponible para el equipo directivo de los establecimientos participantes, contando con reportes a nivel de cursos y a nivel global del colegio. Estos reportes podrían ser un material de insumo relevante para el diagnóstico, seguimiento o evaluación, facilitando la toma de decisiones locales y oportunas desde el equipo directivo, la jefatura de la Unidad Técnico Pedagógica (UTP) y/o los equipos de apoyo psicosocial o psicoeducativo del establecimiento escolar. 


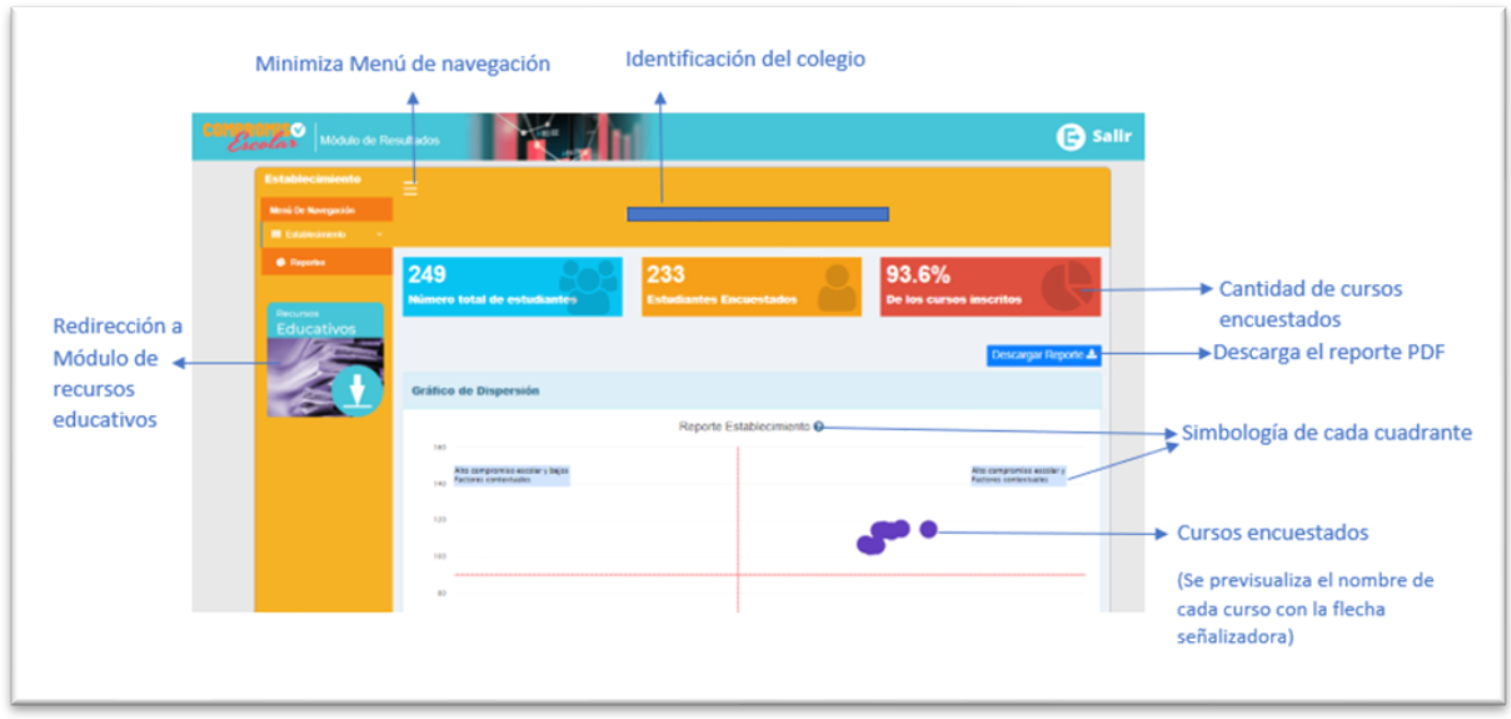

Figura 6. Reportes para el Colegio

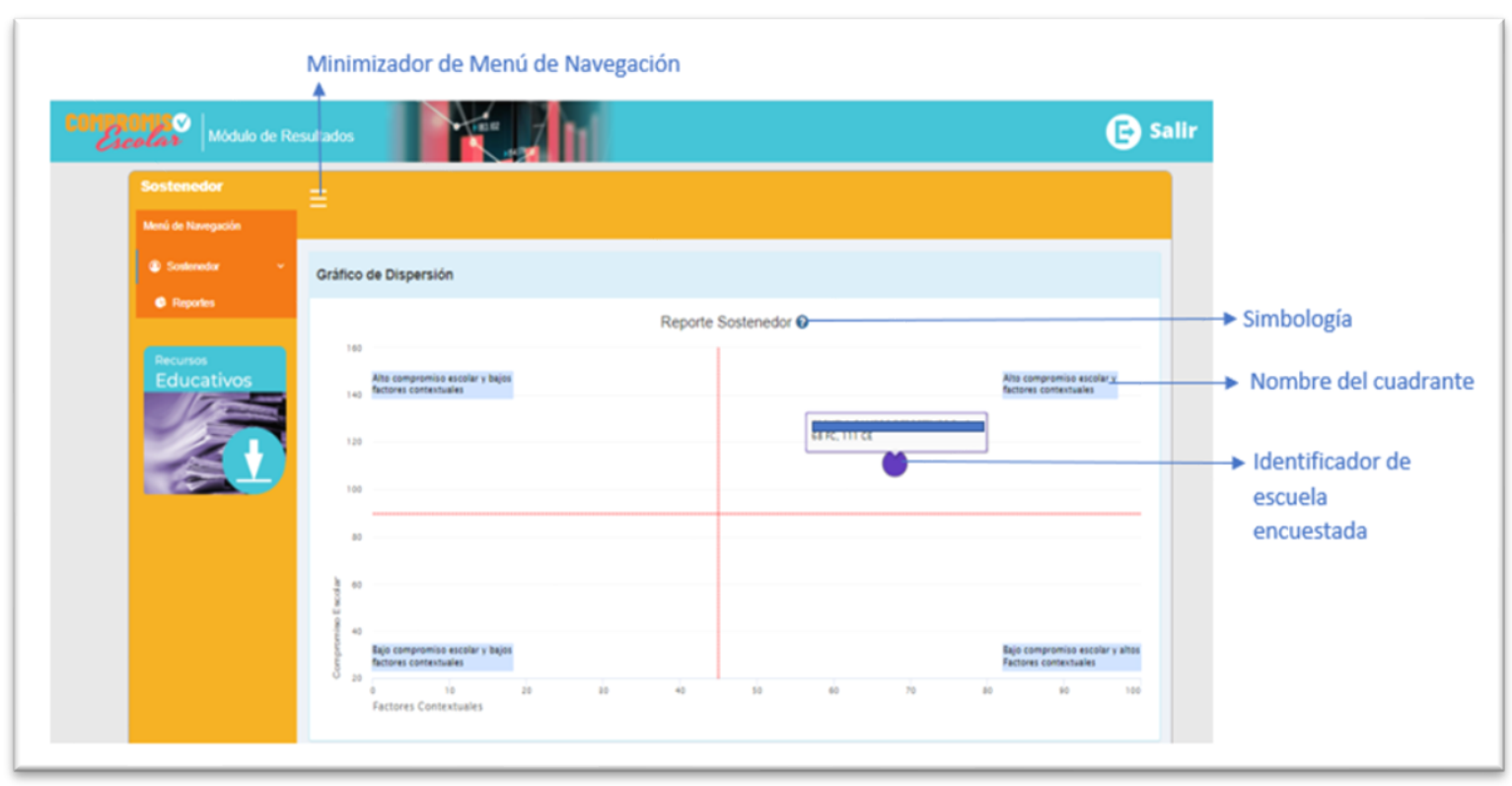

Figura 7. Reportes para el Sostenedor

En este contexto, el tipo de reportes utilizados para presentar la información es del mismo tipo disponible para los/as profesores/as. Sin embargo, en el caso del equipo directivo o los sostenedores (administración municipal, servicio local de educación, otro) tienen la opción de visibilizar reportes de todos los cursos de su colegio, para lo cual inicialmente debe seleccionar el curso a través de un menú pop-up y luego seleccionar el tipo de reporte que quiere revisar (Figura 6). Además, los directivos pueden ver los mismos reportes, pero considerando los valores promedios de todos los estudiantes del colegio. En este caso, en el gráfico de dispersión, cada punto representa el valor promedio de cada uno de los cursos del colegio. A partir de esta información, el equipo directivo se puede formar un panorama global de los distintos cursos del colegio y cómo están aportando al panorama general de su institución.

\section{Reportes para el Sostenedor}

En el caso del sostenedor que agrupa un conjunto de colegios, el módulo de reportes entrega información para el equipo directivo, a nivel de los distintos colegios y a nivel global de la red de colegios.

$\mathrm{Al}$ igual que en el caso de los reportes para el colegio, aquí se utilizan los mismos formatos disponibles para los/as profesores/as. Pero en este caso los reportes disponibles están a nivel de colegios, con los puntajes promedios obtenidos 


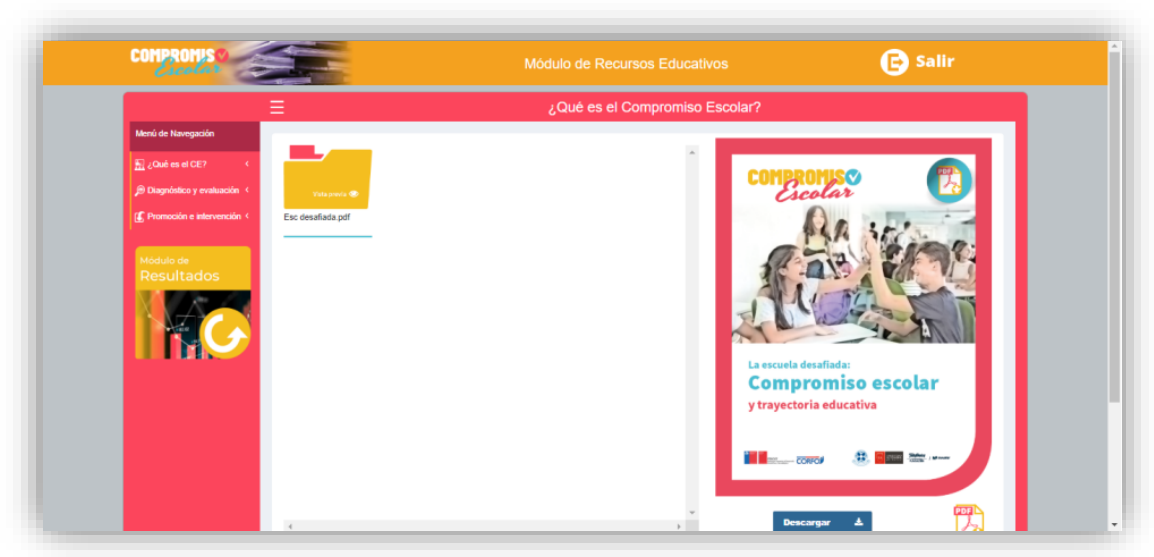

Figura 8. Ejemplo de recurso educativo "Esc. desafiada" (Escuela desafiada) en formato PDF en la sección de ¿Qué es Compromiso escolar? En el Módulo de Recursos educativos

por los estudiantes, y a nivel global del sostenedor, donde se promedian los puntajes de todos los estudiantes de la red de establecimientos que administra el sostenedor.

En el contexto del perfil sostenedor, en caso de querer revisar la situación de algún colegio en particular, puede hacer uso del menú pop-up y luego seleccionar el tipo de reporte que quiere revisar (Figura 7). De esta forma, el perfil puede mostrar los mismos reportes, pero considerando los valores promedios de cada colegio y los valores promedio de la red de colegios administrados por el sostenedor.

En el gráfico de dispersión a nivel global, cada punto representa el valor promedio de cada colegio. A partir de esta información, el equipo directivo del sostenedor se puede formar un panorama global de los distintos colegios que administra y cómo ellos están aportando al panorama global de la red de colegios.

\section{3) Módulo de Apoyo pedagógico}

\section{Módulo con material de apoyo para los profesores}

Este módulo está abierto a la comunidad de usuarios de la plataforma ( $\sin$ acceso personalizado) y posee tres secciones: ¿Qué es el compromiso escolar?; Diagnóstico y Evaluación; y promoción e intervención. En cada sección se pueden descargar de forma gratuita recursos educativos como diapositivas, documentos, audios y videos (ver Figura 8).

\section{Módulos complementarios de soporte Módulo de Autogestión}

En este módulo se realiza el registro de los alumnos con sus datos personales. (Nombre Completo, RUT, ciudad, fecha de nacimiento). A través de la digitalización de su cédula de identidad se generará un código de acceso para que se realice la encuesta de compromiso escolar.

En este módulo se muestran los colegios y cursos generados para la posterior aplicación de la encuesta. También se puede ver el avance de la aplicación de la encuesta en tiempo real y se puede exportar a Excel todos los usuarios creados.

\section{Módulo de Monitoreo de Encuesta}

En este módulo se muestra toda la información de la aplicación de la encuesta en un curso determinado en tiempo real, permitiendo exportar reportes de las encuestas contestadas.

\section{Desde el contexto chileno a las aplicaciones en países de Iberoamérica}

Durante 2019 se recogieron datos en centros educativos participantes de Perú, Colombia, Uruguay y España. Las versiones finales de los cuestionarios fueron alojados para cada país en la plataforma informática de forma diferenciada, permitiendo a cada uno de ellos acceder a la versión del cuestionario de su país (ver Figura 9). De este modo, los cuestionarios fueron los mismos (con directa equivalencia entre ítems), con pequeñas variaciones en la redacción y el vocabulario adaptado a cada país, pero manteniendo la equivalencia en el contenido. 


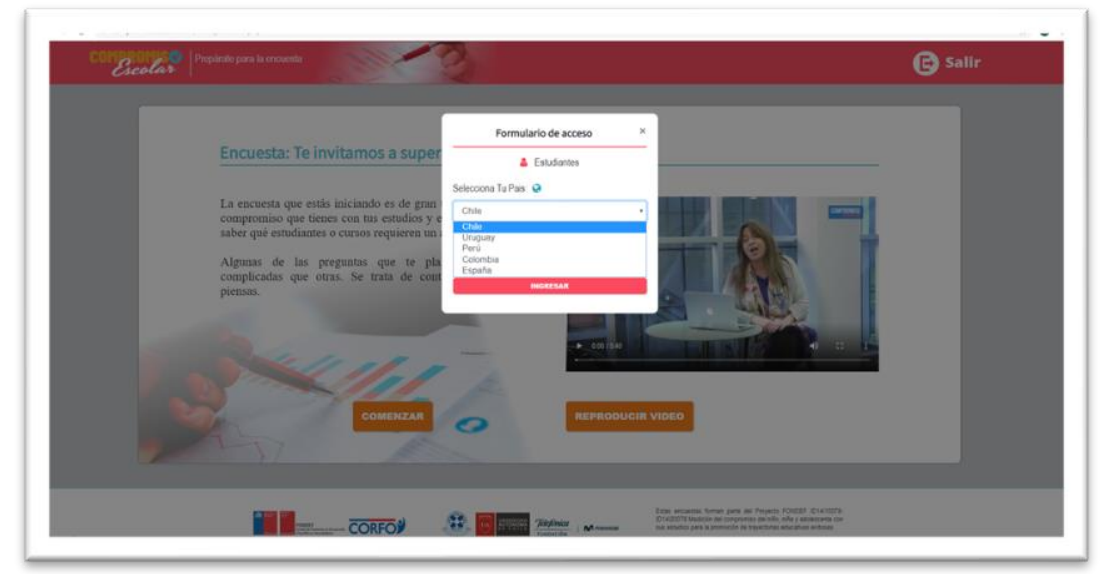

Figura 9. Acceso a los cuestionarios en forma diferenciada por cada país

En todos los países se dieron las mismas indicaciones para la recogida de los datos. En relación con las características de los participantes, estos debían estar matriculados en centros educativos de enseñanza media y básica de extractos socioeconómicos bajos en cursos equivalentes entre los países que abarcaran cinco cursos cuyas edades promedios van desde los 12 a los 16 años. En cuanto a los requerimientos para realizar las evaluaciones online, se indicó que era imprescindible que los centros contaran con computadoras con conexión a internet dado que las evaluaciones debían realizarse online.

Se fijó un tamaño muestral aproximado de 400 estudiantes por país, con el objetivo de permitir realizar los análisis de validación para cada uno (sin diferenciar por cursos) y posteriormente comparaciones entre los mismos. Dada la necesidad de contar con una muestra equiparada en cuanto al sexo, se determinó que se seleccionarían centros mixtos que fueron seleccionados de por conveniencia. En cuanto a los cursos, se indicó que la muestra estuviera equiparada, dando la indicación de conseguir alrededor de 80 estudiantes por cada uno de los cinco cursos objetivo.

La plataforma, el acceso y demás aspectos relacionados con su funcionamiento, fue responsabilidad del equipo chileno, el cual contrató a una empresa para tal efecto, proporcionando los datos necesarios para generar los códigos de accesos para cada estudiante. En cada una de las recogidas de datos estuvo presente el responsable del país participante (o alguna persona que asignaron debidamente capacitada), así como los/as profesores/as del curso, para generar un ambiente confortable y de apoyo a los participantes. En relación con los aspectos éticos, el procedimiento de recogida de datos fue adaptado a los requerimientos de cada país, contando al menos con la autorización de los centros, los consentimientos de los tutores y los asentimientos de los estudiantes.

Se alcanzó un tamaño muestral de 1578 estudiantes que cursaban cursos equivalentes en los cuatro países que participaron: En Colombia se evaluaron 438 estudiantes que cursaban $7^{\circ}, 8$ y $9^{\circ}$ grado de educación básica secundaria, y $10^{\circ}$ y $11^{\circ}$ de educación media. En Uruguay participaron 391 estudiantes que cursaban $1^{\circ}, 2^{\circ}$ y $3^{\circ}$ año del ciclo básico de media y $1^{\circ}$ y $2^{\circ}$ de bachillerato. En España participaron 394 estudiantes que cursaban $1^{\circ}, 2^{\circ}, 3^{\circ}$ y $4^{\circ}$ grado de enseñanza secundaria obligatoria y $1^{\circ}$ de bachillerato. Finalmente, en Perú participaron 355 estudiantes que cursaban $1^{\circ}$, $2^{\circ}, 3^{\circ}, 4^{\circ}$ y $5^{\circ}$ de educación secundaria.

Esto da cuenta del trabajo colaborativo internacional exitoso para el desarrollo de la Plataforma de CE 2.0 y el ajuste del instrumento de medición del CE y los factores contextuales para Chile, Uruguay, Colombia, Perú y España.

\section{Discusión}

Todo estudiante merece vivir en sus años escolares, experiencias enriquecedoras de aprendizaje que estimulen su deseo de comprensión del mundo, y que promuevan el avance de todas las dimensiones de su desarrollo. Merece lograr aprendizajes significativos, pero también sentirse parte de una comunidad educativa que lo acoge, lo respeta, lo escucha y 
genera las condiciones para su crecimiento como aprendiz y como ciudadano. Desafortunadamente, sabemos que no siempre es así. Factores contextuales del alumno pueden predisponerlo al fracaso escolar, pero también en ocasiones se suman a ellos variables de la propia experiencia escolar, que amplifican las dificultades, y conducen a la repitencia y la deserción escolar. Considerando que la escuela es un espacio privilegiado de participación que genera una oportunidad de transmitir aprendizajes y desarrollar actitudes y habilidades (Pérez-Salas, Sirlopú, Cobo, \& Awad, 2018), todo niño o niña que fracasa en ese espacio debería ser considerado como un indicador de las fallas del sistema social y educativo y no del propio alumno.

Por esta razón, contar con indicadores que permitan anticipar con tiempo suficiente el inicio de este proceso, ha sido un objetivo de investigadores educativos por décadas. Entre todos ellos, la medición del nivel de $\mathrm{CE}$ ha destacado como un fuerte predictor de las variables que conducen a trayectorias educativas incompletas o exitosas.

Pero cuando se trata no sólo de evitar que los alumnos deserten del sistema, sino además que permanezcan en él construyendo experiencias de aprendizaje de calidad, se necesita desarrollar formas más completas (pero a la vez más simples) de medir las variables y los factores contextuales asociados a ellas; y eso es lo que se ha intentado en este proyecto de investigación aplicada, con el desarrollo de una versión 2.0 de medición del CE.

La comprensión de la vivencia de un alumno en el sistema escolar, debe abarcar el estudio de los aspectos singulares de la historia de vida de cada individuo, entrelazados con las experiencias de aprendizaje y desarrollo que las instituciones educativas deben ofrecer; y por tanto, el CE no puede ser estudiado sólo desde el logro de estándares y metas académicas homogeneizadas, sino que debe ser entendido como el proceso de construcción individual de un proyecto de vida como alumno y como persona.

Por esta razón, el modelo 2.0 de medición del $\mathrm{CE}$, que aquí se presenta, abarca no sólo la identificación de los estudiantes en riesgo de deserción, sino la identificación, por ejemplo, de aquellos alumnos con alto $\mathrm{CE}$, que requieren estrategias diferenciales de acompañamiento entre ciclos educativos, especialmente en su tránsito hacia la educación superior.

La plataforma desarrollada tiene el potencial de poder ser utilizada por cualquier estudiante, profesor, o directivo, para analizar la información a nivel individual, de curso o por institución, permitiendo realizar un seguimiento longitudinal respecto a cómo se van configurando en el tiempo, los factores contextuales y el CE de una comunidad educativa, como parte de la experiencia escolar compartida por todos los actores del sistema. Otro potencial de la plataforma, desde la imposibilidad de imaginar un mundo sin Internet, se relaciona con la posibilidad de escalar o acercar masivamente el instrumento de medición a las comunidades educativas, debido a que el uso de las plataformas digitales ha crecido de forma exponencial (Castro \& Lupano, 2018).

Desde una perspectiva de inclusión, es deseable reconocer la diversidad de caminos posibles en las trayectorias educativas de los alumnos, desde la óptica de los propios alumnos y sus vivencias. Por eso, aunque la medición del CE puede ser vista como un objetivo en sí mismo, con la utilidad de ser un factor predictor del rendimiento o la permanencia en la escuela, en este proyecto se presenta como una oportunidad (disponible de la manera más sencilla posible) de formación para profesores y directivos, para tomar conciencia de la necesidad de tener mecanismos confiables de monitoreo, de la vivencia de sus alumnos y de su vínculo motivacional y afectivo con la escuela.

La toma de decisiones basada (o al menos "informada") en evidencias, es un principio de gestión de calidad en las normativas ISO 9001 (2015) y un principio orientador obligado para la implementación de intervenciones psicosociales (Drisko \& Grady, 2019). Por esta razón, con base en la sistematización de intervenciones basadas en evidencia (De Toro et al., 2018) queda claro que las instituciones educativas y sus actores, podrán cumplir mejor su función si cuentan a su disposición con medios como esta plataforma, que pongan a su alcance de manera sencilla, la información que les permita tomar decisiones pertinentes y a tiempo.

Queda pendiente la tarea de desarrollar un nuevo módulo tecnológico web que permita 
identificar estrategias específicas adecuadas (de promoción del CE y los factores contextuales asociados), en función del ciclo educativo de los estudiantes y las condiciones particulares de cada contexto escolar. También un módulo de orientaciones particular para cada subtipo de CE (cognitivo, afectivo y/o conductual) y en función de los actores (familia, pares y profesores/as) que muestren mayores necesidades y oportunidades de mejoras en función al contexto de cada establecimiento escolar.

Otro desarrollo pendiente es la parametrización de las fases del proceso de implementación, a través de fichas de registros online e infografías con orientaciones técnicas acerca de: la identificación y selección de estrategias de promoción del CE y los factores contextuales; el registro del plan de trabajo acordado por los actores claves en función de los resultados arrojados, el seguimiento y monitoreo de las estrategias seleccionadas y los indicadores de alerta por estudiante, curso y establecimiento escolar.

\section{Financiamiento}

Este artículo ha recibido el apoyo de los proyectos ANID /FONDECYT 1210172, FONDEF ID14I20078 y FONDEF IT 19 I0012 de la Comisión Nacional de Investigación Científica y Tecnológica, CONICYT, y CORFO 18ISV93400 del Ministerio de Economía, Fomento y Turismo de Chile.

\section{Referencias}

Ansong, D., Okumu, M., Bowen, G., Walker A., \& Eisensmith, S. (2017). The role of parent, classmate, and teacher support in student engagement: Evidence from Ghana. International Journal of Educational Development, 54, 51-58. doi:10.1016/j.ijedudev.2017.03.010

Archambault, I., Janosz, M., Fallu, J.-S., \& Pagani, L. S. (2009). Student engagement and its relationship with early high school dropout. Journal of Adolescence, 32(3), 651670. doi:10.1016/j.adolescence.2008.06.007

Ashwin P., \& McVitty D. (2015). The meanings of student engagement: Implications for policies and practices. En A. Curaj, L. Matei, R. Pricopie, J. Salmi, y P. Scott (Eds). The European higher education area: Between critical reflections and future policies (pp. 343-359). Springer.

doi: 10.1007/978-3-319-20877-0_23

Bowles, S., Mindee, O., Heppen, J., Yerhot, L., Scala J., \& Perry, M. (2013). Middle grades early warning intervention monitoring system implementation guide. National High School Center. Washington DC: American Institud For Research.

Castro, A., \& Lupano, M. L. (2018). Perfiles diferenciales de usuarios de internet, factores de personalidad, rasgos positivos, síntomas psicopatológicos y satisfacción con la vida. Revista Iberoamericana de Diagnóstico y Evaluación - e Avaliação Psicológica, 53(4), 79-90.

Christenson, S. L., Reschly, A. L., Appleton, J. J., Berman- Young, S., Spanjers, D. M., \& Varro, P. (2008). Best practices in fostering student engagement. En A. Thomas y J. Grimes (Eds.), Best practices in school psychology V, vol. 6 (pp. 1099-1120). National association of School Psychologists.

Christenson, S. L., Reschly, A. L., \& Wylie, C. (2012). Handbook of research on student engagement. New York: Springer.

Csikszentmihalyi, M., \& Shernoff, D. J. (2009). Cultivation engaged learners and optimal learning environment. En R. Gilman, S. E. Huebner y M. J. Furlong (Eds.), Handbook of positive psychology in schools (pp. 131-145). New York NY: Routledge.

De Toro. X., Saracostti, M., Lara, L., Mayorga, C., Navarro, J. J., Riquelme, S., Sandoval, M. J., Trigger, J., Viveros, J., \& Yáñez, M. T. (2018). Manual de intervenciones sobre el compromiso escolar. Variable Clave para Predecir Procesos de Desescolarización. Producto del proyecto FONDEF ID14I10078 medición del compromiso del niño, niña y adolescente con sus estudios para la promoción de trayectorias educativas exitosas. Universidad de la Frontera. Recuperado de http://humanidades.ufro.cl/images/libros/ORG _Manual_de_intervenciones.pdf 
Drisko, J., \& Grady, M. (2019). Evidence Based Practice in Clinical Social Work, 2nd edition. New York: Springer International.

Fernández-Zabala, A., Goñi, E., Camino, I., \& Zulaika, L. M. (2016). Family and school context in school engagement. European Journal of Education and Psychology, 9(2), 47-55. doi:10.1016/j.ejeps.2015.09.001

Fredricks, J. A., Filsecker, M., \& Lawson, M. A. (2016). Student engagement, context and adjustment: Addressing definitional, measurement and methodological issues. Learning and Instruction, 43, 1-4. doi:10.1016/j.learninstruc.2016.02.002

Fredricks, J. A., Reschly, A. L., \& Christenson, S. L. (2019). Handbook of Student Engagement Interventions. 1st Edition Working with Disengaged Students. Academic Press.

González González, M. T. (2010). El alumno ante la escuela y su propio aprendizaje: Algunas líneas de investigación en torno al concepto de implicación. Revista Iberoamericana sobre Calidad, Eficacia y Cambio en Educación, 8(4), 10-31. Recuperado de https://revistas.uam.es/reice/article/view/4735/ 5169

International Organization for Standardization. (2009). ISO 31000:2009: Gestión del riesgo Principios y directrices. Ginebra: ISO.

Jimerson, S. R., Renshaw, T. L., Stewart, K., Hart, S., \& O’Malley, M. (2009). Promoting school completion through understanding school failure: A multi-factorial model of dropping out as a developmental process. Romanian Journal of School Psychology, 1(9), 7-29.

Miranda-Zapata, E., Lara, L., Navarro, J.-J., Saracostti, M., \& de-Toro, X. (2018). Modelización del efecto del Compromiso Escolar sobre la Asistencia a clases y el Rendimiento escolar en una muestra de estudiantes chilenos. Revista de Psicodidáctica, 23(2), 102-109. doi:10.1016/j.psicod.2018.02.003

Pérez-Salas, C., Sirlopú, D., Cobo, R., \& Awad, A. (2018). Análisis bifactorial de la Escala de Participación Escolar en una muestra de estudiantes Chilenos. Revista Iberoamericana de Diagnóstico y Evaluación - e Avaliação Psicológica, 52(3), 27-39.
Quin, D., Heerde, J., \& Toumbourou, J. (2018). Teacher support within an ecological model of adolescent development: Predictors of school engagement. Journal of School Psychology, 69, 1-15. doi:10.1016/j.jsp.2018.04.003

Roundfield, K., Sánchez, B., \& McMahon, S. (2016). An ecological analysis of school engagement among urban, Low-Income Latino Adolescents. Youth and Society, 50(7), 905-925. doi:10.1177/0044118X16639986

Saracostti, M., Lara, L., \& Miranda-Zapata, E. (2019). Plataforma tecnológica de evaluación del compromiso Escolar, factores de contexto y reporte de resultados online: Breve Reporte Técnico. Electronic Journal of Research in Educational Psychology, 17(1), 193-212. doi:10.25115/ejrep.v17i47.2049

Wang, M.-T., \& Fredricks, J. A. (2014). The reciprocal links between school engagement, youth problem behaviors, and school dropout during adolescence. Child Development, 85(2), 722-737. doi:10.1111/cdev.12138

Zaff, J. F., Donlan, A., Gunning, A., Anderson, S. E., McDermott, E., \& Sedaca, M. (2017). Factors that promote high school graduation: A review of the literature. Educational Psychology Review, 29(3), 447-476. doi:10.1007/s10648-016-9363-5 